\title{
ESTUDO REOLÓGICO DE UM FLUIDO DE PERFURAÇÃO COM CARACTERÍSTICAS BIODEGRADÁVEIS A BASE DE TENSOATIVO NP40 E ÓLEO DE CANOLA
}

\author{
A. I. C. GARNICA ${ }^{1}$, A. S. L. SOARES ${ }^{2}$, F. D. DA S. CURBELO ${ }^{3}$ \\ Universidade Federal da Paraíba \\ ORCID ID: http://orcid.org/0000-0001-9600-5110 \\ alfredocurbelo@yahoo.com ${ }^{1}$ \\ Artigo submetido em 18/02/2020 e aceito em 21/08/2020
}

DOI: $10.15628 /$ holos.2020.9571

\section{RESUMO}

A perfuração de poços é um dos pilares na exploração de petróleo, é o elo entre a superfície e o reservatório, e uma parte vital da perfuração é o fluido utilizado que deve se mostrar eficiente no processo. $\mathrm{O}$ objetivo deste trabalho foi desenvolver um fluido de base microemulsionada aplicável à perfuração de poços de petróleo que se destaca por ser biodegradável. A partir da construção de diagramas de fases ternários, foi possível a investigação e obtenção de um ponto de microemulsão, formado por três componentes, uma fase oleosa (óleo de canola), uma fase aquosa (solução aquosa de $2 \mathrm{KCl}$ em peso) e um tensoativo não iônico (NP40), que foi utilizado como base para o fluido, e em seguida foi aditivado para a agregação de características desejáveis para perfuração. Foi formular dois fluidos de perfuração e, com isso, foram obtidas suas respectivas curvas de fluxo, para determinar a classificação e o modelo reológico que melhor se ajustou com base na Norma API 13B (2012). A densidade e o volume de filtrado também foram determinados. Os fluidos obtidos apresentaram aspecto homogêneo, com massas específicas de 1,14 e $1,19 \mathrm{~g} / \mathrm{cm}^{3}$, volume de filtrado de, apenas, $6,4 \mathrm{~mL}$ para um fluido e 12,6 para o outro. Os fluidos possuíam viscosidade aparente entre 115 e 136,8 cP, viscosidade plástica entre 104,5 e 125 cP, e limite de escoamento entre 21 e $23,5 \mathrm{~N} / \mathrm{m}^{2}$. O modelo reológico de Herschel-Bulkley ajustou melhor os dados experimentais. Foi realizado um teste de compatibilidade entre cada fluido e uma pasta de cimento para estudo da contaminação, mostrando uma compatibilidade satisfatória.

PALAVRAS-CHAVE: óleo de canola, microemulsão, fluido de perfuração, compatibilidade.

\section{RHEOLOGICAL STUDY OF A DRILLING FLUID WITH BIODEGRADABLE FEATURES BASED ON NP4O SURFACE AND CANOLA OIL}

\begin{abstract}
Well drilling is one of the pillars in oil exploration, it is the link between the surface and the reservoir, a vital part of the drilling is the fluid used that must be efficient in the process. The objective of the work is to develop a microemulsion based fluid applicable to the drilling of oil wells that stands out for being biodegradable. From the construction of ternary phase diagrams, it was possible to investigate and obtain a microemulsion point formed by three components, one in the oil phase, one in the water phase and a surfactant that was used as the base for the fluid, which was then additive for the aggregation of desirable characteristics for drilling. Viscosity measurements were made on Fann $35 \mathrm{~A}$, used to determine which classification and rheological model best fit this behavior based on API 13B (2012). Density was measured on the Fann 140 mud scale, the filtrate test
\end{abstract}

on the API Pressurized Filter Press. The fluids obtained have a homogeneous appearance with specific masses of 1.14 and $1.19 \mathrm{~g} / \mathrm{cm}^{3}$, the volume of filtrate obtained after a period of $30 \mathrm{~min}$ at a pressure of 100 psi was only $6.4 \mathrm{~mL}$ for a fluid and 12,6 for the other, the fluids have an apparent viscosity of 115 and $136.8 \mathrm{cP}$ and the plastic viscosity of 104.5 and $125 \mathrm{CP}$, yield limit of 21 and $23.5 \mathrm{~N}$ $/ \mathrm{m}^{2}$, from the consistency curve obtained through the graph plotted between the Shear Stress $(\tau)$ in N / $\mathrm{m}^{2}$ and the strain rate $(\gamma)$ in $1 / \mathrm{s}$, the parameters and the adjustment to a Herschel-Bulkley rheological model were obtained, being a power fluid with initial flow limit, the fluid is non-Newtonian. A compatibility test was carried out with a cement paste to study the contamination, the compatibility was satisfactory.

KEYWORDS: canola oil, microemulsion, drilling fluid, compatibility. 


\section{INTRODUÇÃO}

Durante séculos, o homem vem desenvolvendo novas formas para aprimorar os métodos de perfuração de poços (óleo, gás e água). Com o decorrer dos anos, surgem novos avanços nos campos da engenharia mecânica, civil, química, entre outras, que vão servir como base para o surgimento de métodos mais eficientes e dinâmicos para a perfuração de poços, principalmente os poços de petróleo. Um exemplo muito ilustrativo desses avanços é o surgimento dos métodos rotacionais de perfuração que conferem uma maior eficiência e rapidez na perfuração (Seixas, 2010).

Enquanto uma bomba alternativa funciona como o coração do sistema de perfuração rotativo, o escoamento do fluido de perfuração se assemelha ao fluxo sanguíneo. A bomba transmite a potência hidráulica necessária para deslocar o fluido através do poço e auxiliar na perfuração, por meio da ação da broca. O fluido é constituído com os ingredientes necessários para manter o poço "saudável" (Ribeiro, 2013).

Atualmente, a etapa de perfuração é capaz de atingir regiões de grande complexidade. $\mathrm{Na}$ perfuração rotativa, as rochas são perfuradas pela ação da rotação e peso aplicados à broca, que se localiza no fim da coluna de perfuração. Os pedaços rochosos são removidos através da ação contínua de um fluido de perfuração. O fluido é injetado por bombas para o interior da coluna de perfuração e retorna à superfície através do espaço anular formado pelas paredes do poço e pela coluna (Thomas, 2004).

Desta forma, a microemulsão apresenta-se como um potencial constituinte de fluidos de perfuração, uma vez que diversos autores, tais como Son (1973), Davies et al. (1997), Cunha Filho (2015), Sousa (2018) e Magalhães (2018) citam os benefícios da utilização desse sistema. Dentre os benefícios citados pode-se destacar a contribuição para uma maior estabilidade do fluido, quando comparado ao mesmo preparado a partir de emulsões, o que proporciona a vantagem de manter os cascalhos em suspensão por mais tempo durante as paradas do poço, além de serem resistentes à corrosão, terem boas características de lubricidade, formarem reboco menos permeável e fino e possuir baixa tensão interfacial. A microemulsão ainda permite a utilização da fase oleosa composta por um óleo vegetal, promovendo uma imediata redução nas características prejudiciais ao meio ambiente o que acrescenta a tal tipo de fluido uma maior sustentabilidade.

Os sistemas microemulsionados, por serem estáveis termodinamicamente, espontâneos (Silva, 2011) e facilmente preparáveis, tem uma vasta aplicação na indústria. O uso desses sistemas em fluidos de perfuração foi estudado por alguns pesquisadores, como Hayes, Haws e Gogarty (1977), Davies, Meeten e Way (1997) e Quintero et al. (2009), em que apresentaram vantagens de estabilidade em uma ampla faixa de condições salinas.

Embora ainda pouco estudadas no cenário atual, as microemulsões apresentam um grande potencial para base de fluidos de perfuração, uma vez que consegue introduzir várias propriedades desejáveis, a mais latente delas, é a utilização de compostos biodegradáveis que atendem a determinações ambientais cada vez mais preocupadas com esse tipo de fluido. 


\section{REVISÃO BIBLIOGRÁFICA}

\subsection{Fluido de perfuração}

Os fluidos de perfuração são composições destinadas a auxiliar o processo de perfuração de poços de petróleo, poços tubulares e operações de sondagem (Amorin, 2003). Quando líquidos, os fluidos de perfuração são misturas complexas que podem conter água, material orgânico, sais dissolvidos e sólidos em suspensão nas mais variadas proporções (Campos, 2007).

Quando o principal constituinte é um líquido (água ou óleo), neste caso, tem-se um fluido à base de água ou à base de óleo. Quando a água e o óleo estão presentes, forma-se uma emulsão com agitação e a adição de um agente emulsificante. A natureza química do emulsificante determina se o óleo é emulsificado na água (emulsão de óleo, ou seja, a água é a fase contínua) ou se a água é emulsificada no óleo (emulsão inversa, ou seja, o óleo é a fase contínua).

Segundo a American Society of Mechanical Engineers (ASME, 2005), fluidos de perfuração tem a função de auxiliar no resfriamento da broca de perfuração, bem como em sua limpeza e lubrificação, auxiliar no carreamento dos cascalhos até a superfície e também mantê-los suspensos caso haja paradas no processo de bombeamento, impedindo que o seu acúmulo no poço, dar maior estabilidade às paredes do poço, garantindo que não haja desmoronamento, bem como evitar a interação química entre a rocha e a coluna de perfuração, auxiliar no controle do influxo de fluidos (kick) para dentro do poço através da pressão hidrostática que exercem durante a operação.

Para isso, os fluidos de perfuração recebem aditivos que lhes garantem propriedades de densidade ou peso, o que Ihes conferem uma maior pressão hidrostática dentro do poço, bem aditivos que permitem a formação de um reboco fino e de baixa permeabilidade, que controla o volume de filtrado perdido para a formação.

As propriedades reológicas relacionam-se com as características de escoamento do fluido sob várias condições de fluxo. Em geral, fluidos de perfuração se comportam como fluidos nãoNewtonianos, sendo necessário se estabelecer a relação entre a taxa de cisalhamento $\gamma$ (diferencial da velocidade do fluido ao longo de uma trajetória) e a tensão de cisalhamento $\tau$ de fluidos não newtonianos depende da composição do fluido e pode ser disposta em gráficos chamados curvas de consistência ou modelo de fluxo como na Figura 1 (Caenn, Darley \& Gray, 2011; Machado, 2002).

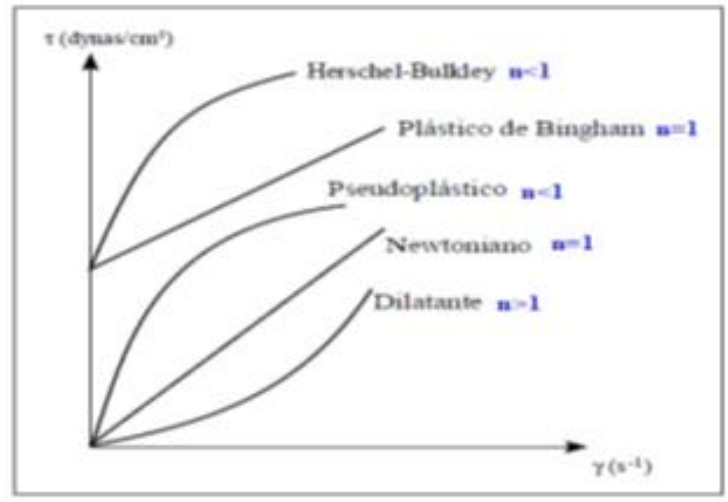

Figura 1: Curvas de consistência associadas ao parâmetro $n$. 
O modelo mais completo ao qual se adequa a fluidos de perfuração, é o que une a característica de fluido de potência com o de Limite de escoamento, que basicamente é a junção dos modelos de Fluido Pseudoplástico com o Plástico de Bingham, e é chamado de Modelo de Herschel-Bulkley, que é tido como mais completo por envolver três parâmetros reológicos, sendo eles: $\tau_{0}$, denominado de limite de escoamento inicial, que permite prever a força mínima requerida para iniciar o fluxo; $k$, denominado de parâmetro de consistência, que indica o grau de resistência do fluido diante do escoamento, expresso em $\mathrm{Nm}^{-2} \mathrm{~s}^{n}$ ou Pa.s ${ }^{n}$ (SI); e $\mathrm{n}$, denominado de índice de comportamento, que indica fisicamente o afastamento do fluido do modelo newtoniano, sendo uma grandeza adimensional (Machado, 2002; Shiroma, 2012). A Equação 2.1 que define o modelo é mostrada abaixo.

$\tau=\tau_{0}+k \gamma^{n}$

Para determinar os parâmetros deste modelo, inicialmente estima-se o valor de $\tau_{0}$ por extrapolação através do gráfico de tensão cisalhante $(\tau)$ versus taxa de deformação $(\gamma)$ em coordenadas cartesianas, posteriormente, determina-se o valor de $k$ e $n$ através do gráfico de ( $\tau$ $\tau_{0}$ ) versus $\gamma$, em coordenadas logarítmicas (Machado, 2002).

\section{METODOLOGIA}

Para os ensaios reológicos, foram utilizados dois fluidos de perfuração com base microemulsionada, base essa composta por óleo de canola (FO), solução aquosa $2 \% \mathrm{KCl}(\mathrm{FA})$ e tensoativo não iônico NP40 (T). Sendo o fluido FP1 formado por uma base microemulsionada composta por uma proporção mássica de 15\% FO, 15\% FA e 70\% T, e o fluido FP2 formado por uma base microemulsionada de 50\% FO, 10\% FA e 40\% T. A composição dos fluidos é mostrada na Tabela 1.

Tabela 1: Composição dos fluidos de perfuração.

\begin{tabular}{c|c|c}
\hline Aditivos & Função & Massa (g) \\
\hline Microemulsão & Base do fluido & 300 \\
Argila Closite 20A & Viscosificante & 4 \\
Calcário fino & Obturante & 1 \\
Hidroxipropilamido (HPA) & Controlador de Filtrado & 1 \\
Goma Xantana & Viscosificante & 1 \\
Baritina & Adensante & 40 \\
Cloreto de Potássio & Inibidor & $<1$ \\
\hline
\end{tabular}

Os fluidos foram preparados utilizando o agitador mecânico Hamilton Beach (modelo HMD200), inicialmente são preparados $300 \mathrm{~g}$ da base microemulsionada, que é homogeneizada durante 10 minutos numa rotação de 17.000 rpm, em seguida cada aditivo foi adicionado, um por vez, durante um tempo de agitação de 10 minutos entre eles. A adição de cada material obedeceu a seguinte ordem: base microemulsionada (ME), Argila Closite 20A, Calcário fino, HPA, Goma Xantana e, por último, adensante Baritina.

Foram efetuadas as medidas conforme as normas API 13B-1 2017 e API 13B-2 2014 de viscosidade no viscosímetro Fann 35 A (Figura 2), utilizado para medir a viscosidade aparente (VA) 
e plástica (VP), o limite de escoamento (LE), medidas de gel inicial $\left(G_{0}\right)$ e gel final $\left(G_{F}\right)$ do fluido de perfuração. Além disso, a partir dessas leituras, foi possível estudar o comportamento do fluido e determinar qual classificação e modelo melhor se ajustam a esse comportamento. O objetivo dos testes de gel final e gel inicial foi avaliar a capacidade que o fluido tem em manter os cascalhos em suspensão quando a perfuração é interrompida.

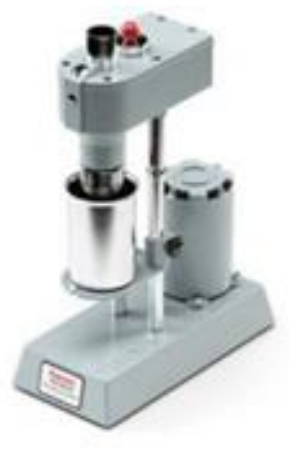

Figura 2: Viscosímetro Fann 35A.

As leituras foram efetuadas para as seguintes velocidades rotacionais 600, 300, 200, 100, 6 e $3 \mathrm{rpm}$, permanecendo o fluido $1 \mathrm{~min}$ em cada velocidade para, em seguida, ser feita a leitura da deflexão. Foram feitos ensaios reológicos a temperatura ambiente, $26^{\circ} \mathrm{C}\left(79{ }^{\circ} \mathrm{F}\right)$. A partir das leituras das deflexões, $\theta$, pôde-se calcular as tensões de cisalhamento, $\tau\left(\mathrm{N} / \mathrm{m}^{2}\right)$, com suas respectivas velocidades de rotação, $N$ (rpm), convertidas para taxa de cisalhamento $\gamma\left(\mathrm{s}^{-1}\right)$ conforme as equações (3.1) e (3.2)

$\tau=0,51 x \theta$

$\gamma=1,703 x N$

Os valores obtidos de tensão de cisalhamento $(\tau)$ e taxa de deformação $(\gamma)$ são plotados a fim de verificar o comportamento do fluido de perfuração.

Outra importante propriedade que necessita ser avaliada no modelo reológico é a viscosidade $(\mu)$ mostrada na Equação (3.3), que apresenta variação quando submetida à taxa de deformação, essa avaliação é feita a partir do gráfico plotado entre essas duas grandezas.

$$
\mu=300 \times \frac{\theta}{N}
$$

Por fim, com o objetivo de se avaliar o modelo reológico, os dados experimentais são submetidos a um modelo de três parâmetros, que é o mais comumente utilizado para fluidos de perfuração segundo, segundo Machado (2002) e Shiroma (2012), conforme a Equação (2.1).

A viscosidade aparente (VA) pode ser calculada dividindo a leitura à $600 \mathrm{rpm}$ por 2 e a viscosidade plástica (VP) pela subtração da leitura a $600 \mathrm{rpm}$ pela leitura a $300 \mathrm{rpm}$, ambas na unidade centiPoise (CP). O limite de escoamento (LE) é dado pela leitura da deflexão à $300 \mathrm{rpm}$ 
subtraído da viscosidade plástica, conforme a Norma API 13B (2012) para viscosímetro Fann. Todas essas propriedades são mostradas nas Equações 3.4, 3.5 e 3.6.

$$
\begin{aligned}
& \mathrm{VA}=\frac{L_{600}}{2} \quad(\mathrm{cP}) \\
& \mathrm{VP}=L_{600}-L_{300} \quad(\mathrm{cP}) \\
& \mathrm{LE}=L_{300}-V P \quad\left(\mathrm{lbf} / 100 \mathrm{ft}^{2}\right)
\end{aligned}
$$

Para o gel inicial $\left(G_{0}\right)$, o tempo de repouso, antes de se iniciar o cisalhamento, é de $10 \mathrm{~s}$, e gel final $\left(G_{F}\right)$ é de 10 min. Para a medição, o fluido é colocado numa rotação de $600 \mathrm{rpm}$ no viscosímetro durante $1 \mathrm{~min}$, então muda-se para $3 \mathrm{rpm}$ e desliga-o. Espera-se o tempo de repouso indicado para cada uma das forças géis e, em seguida, o viscosímetro é ligado e é feita a leitura da máxima deflexão.

Com o objetivo de avaliar a degradação dos fluidos, um ensaio de envelhecimento foi realizado em uma estufa Roller Oven da Fann (Figura 3), em uma célula de pressão de aço inoxidável com capacidade para $260 \mathrm{~mL}$, à temperatura de $66^{\circ} \mathrm{C}\left(151^{\circ} \mathrm{F}\right)$ por $16 \mathrm{~h}$. Após esse período de tempo, o ensaio reológico é novamente realizado para verificar se o fluido sofreu alterações nas suas propriedades com o efeito do tempo, agitação e temperatura.

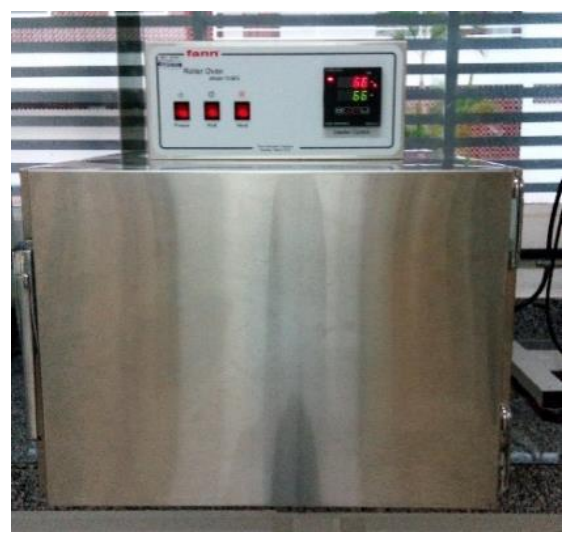

Figura 3: Estufa Roller Oven Fann.

Compatibilidade é a propriedade de dois ou mais fluidos que, quando misturados em diferentes proporções, não apresentam reações químicas ou físicas indesejáveis (Campos, 2005). 0 teste de compatibilidade entre a pasta de cimento padrão (PC) e cada fluido de perfuração (FP1 e FP2) foi realizado conforme a norma API Spec 10A (2004).

Os fluidos de perfuração foram inicialmente homogeneizados por 15 minutos no agitador Hamilton Beach, para manter a estabilidade durante o ensaio. A pasta de cimento e os fluidos de perfuração foram, em seguida, homogeneizados separadamente em um consistômetro atmosférico Chandler Modelo 1200, Figura 4, durante 20 minutos na temperatura de $192^{\circ} \mathrm{C}\left(89^{\circ} \mathrm{F}\right)$. 


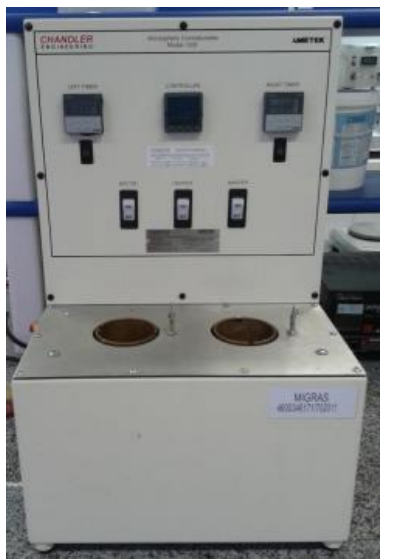

Figura 4: Consistômetro Chandler modelo 1200.

Em seguida, os fluidos foram misturados em diferentes proporções volumétricas, mostradas na Tabela 2, em uma proveta plástica de $500 \mathrm{~mL}$ com o auxílio de uma espátula até que a mistura se tornasse homogênea, com o objetivo de se avaliar a reologia e curvas de fluxo dessa mistura PC/FP1 e PC/FP2.

Tabela 2: Composição de amostras para teste de compatibilidade norma API Spec 10.

\begin{tabular}{c|c|c}
\hline Amostra & PC/FP1 (\%) & PC/FP2 (\%) \\
\hline M1 & $95 / 5$ & $95 / 5$ \\
M2 & $75 / 25$ & $75 / 25$ \\
M3 & $50 / 50$ & $50 / 50$ \\
M4 & $25 / 75$ & $25 / 75$ \\
M5 & $5 / 95$ & $5 / 95$ \\
\hline
\end{tabular}

\section{RESULTADOS E DISCUSSÃO}

Os resultados obtidos para os parâmetros reológicos dos fluidos estão apresentados na Tabela 3.

Tabela 3: Composição de amostras para teste de compatibilidade norma API Spec 10.

\begin{tabular}{c|c|c|c|c|c}
\hline Fluido & VA (cP) & VP $(\mathrm{cP})$ & $\mathrm{LE}\left(\mathrm{lbf} / 100 \mathrm{ft}^{2}\right)$ & $\boldsymbol{G}_{\mathbf{0}}\left(\mathrm{lbf} / 100 \mathrm{ft}^{2}\right)$ & $\boldsymbol{G}_{\boldsymbol{F}}\left(\mathrm{lbf} / 100 \mathrm{ft}^{2}\right)$ \\
\hline FP1 & 115 & 104,5 & 21 & 13 & 15 \\
FP1 & 145,5 & 110,5 & 70 & 12 & 15 \\
Envelhecido & 136 & 125 & 23,5 & 13 & 16 \\
FP2 & 135 & 121 & 28 & 8 & 10 \\
FP2 & 135 \\
Envelhecido & & & &
\end{tabular}

Os fluidos FP1 e FP2 apresentaram valores de viscosidade aparente (VA) e de viscosidade plástica (VP) dentro da faixa de aceitação, de acordo com a norma PETROBRAS N-2604 (1998). A alta viscosidade pode ser explicada pela formulação dos fluidos, que no caso do FP1 apresenta uma alta concentração do tensoativo Ultranex NP 40, e, no caso do FP2, uma alta concentração de óleo de canola. 
Os valores de limite de escoamento (LE) e forças géis obtidos se enquadram também nas normas vigentes para fluidos com esse tipo de formulação, e em comparação com a literatura de acordo com Sousa (2018) e Gonçalves (2018), se assemelha com outros fluidos estudados por outros autores.

O limite de escoamento que indica a resistência ao fluxo, segundo Gray et al. (1988), expressa o valor mínimo de tensão cisalhante que deve ser aplicado ao fluido para que este inicie o escoamento, sendo assim é possível avaliar que o FP1 apresentou uma valor de (LE) consideravelmente menor do que o FP2, e haverá menor resistência ao escoamento inicial, entretanto, ambos os valores estão dentro da faixa aceitável da norma PETROBRAS N-2604 (1998) para este parâmetro e está condizente com os fluidos considerados base óleo encontrados nas literaturas estudadas, tanto em Sousa (2018) e Magalhães (2018).

As medidas da força gel inicial, como pode ser visto as leituras dos fluidos FP1 e FP2, apresentaram valores dentro da faixa de aceitação, e a diferença entre a tensão de reinicio de escoamento entre os tempos de 10 segundos e 10 minutos estão próximos um do outro, indicando que o fluido não se gelifica com o tempo, e assim não necessita de uma maior tensão para que ele reinicie o escoamento.

Os resultados da Tabela 3 apresentam, também, os parâmetros reológicos dos fluidos FP1 e FP2 após o envelhecimento. É possível notar que existe um aumento na viscosidade plástica e viscosidade aparente do fluido FP1, comportamento esse que pode ser explicado pela degradação do fluido durante o ensaio, as forças géis $G_{0}$ e $G_{F}$ para o FP1 se mantiveram praticamente iguais, enquanto que para o FP2 apresentam uma leve diminuição, contudo, todos os valores obtidos se enquadram nos valores de referência descritos nas normas, indicando que o envelhecimento não causou grandes mudanças.

A partir das leituras realizadas no Viscosímetro Fann, foi possível obter a curva de consistência, que relaciona a tensão de cisalhamento $\tau\left(\mathrm{N} / \mathrm{m}^{2}\right)$ e a taxa de deformação $\gamma\left(\mathrm{s}^{-1}\right)$, na qual será possível a obtenção do modelo reológico, de acordo com a Figura 5 e 6, para o fluido FP1 e, também, na condição de envelhecimento.

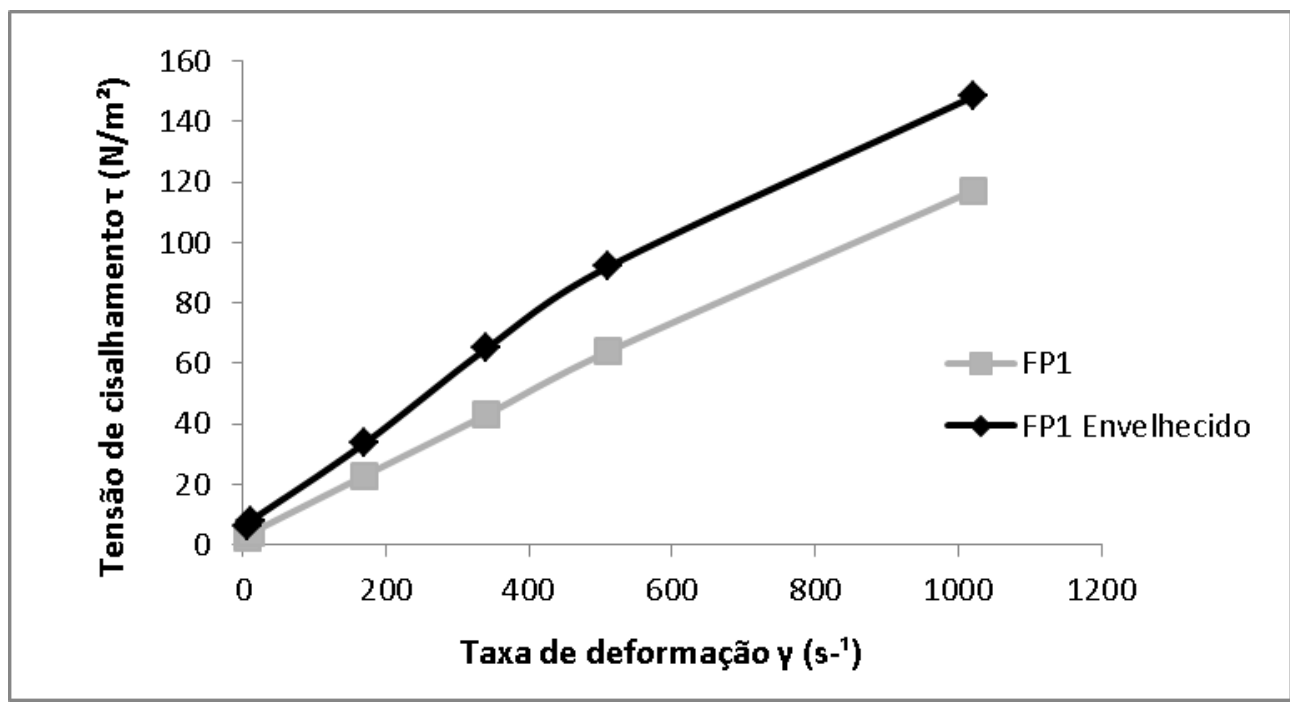

Figura 5: Comportamento reológico da tensão de cisalhamento FP1 e FP1 envelhecido. 
Para a classificação do fluido, também é importante à associação da taxa de cisalhamento com a variação de viscosidade desse fluido, com intuito de investigar o comportamento de fluidos não Newtonianos, a relação entre taxa de cisalhamento e viscosidade é apresentada na Figura 6.

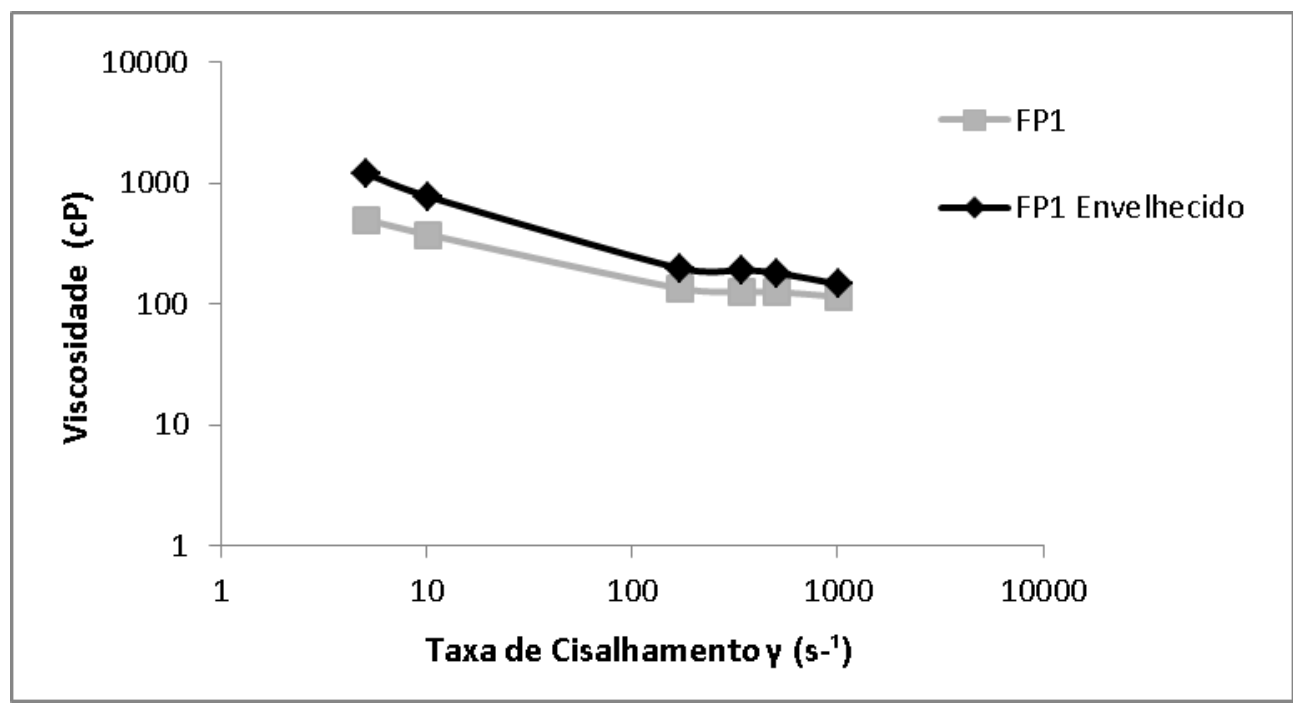

Figura 6: Comportamento reológico da viscosidade FP1 e FP1 envelhecido.

É possível classificar o fluido de perfuração FP1 e FP1 envelhecido como um fluido de comportamento não newtoniano, com características pseudoplásticas, verificadas através da diminuição da viscosidade com o aumento da taxa de cisalhamento, aspecto desejável em fluidos de perfuração conforme a Figura 6.

Para o fluido FP2 e FP2 envelhecido, os resultados estão apresentados na Figura 7, e é possível identificar, assim como no FP1, o comportamento não newtoniano, explicado pela tensão de corte inicial $\tau_{0}$ da curva de consistência, através do comportamento reológico da tensão de cisalhamento.

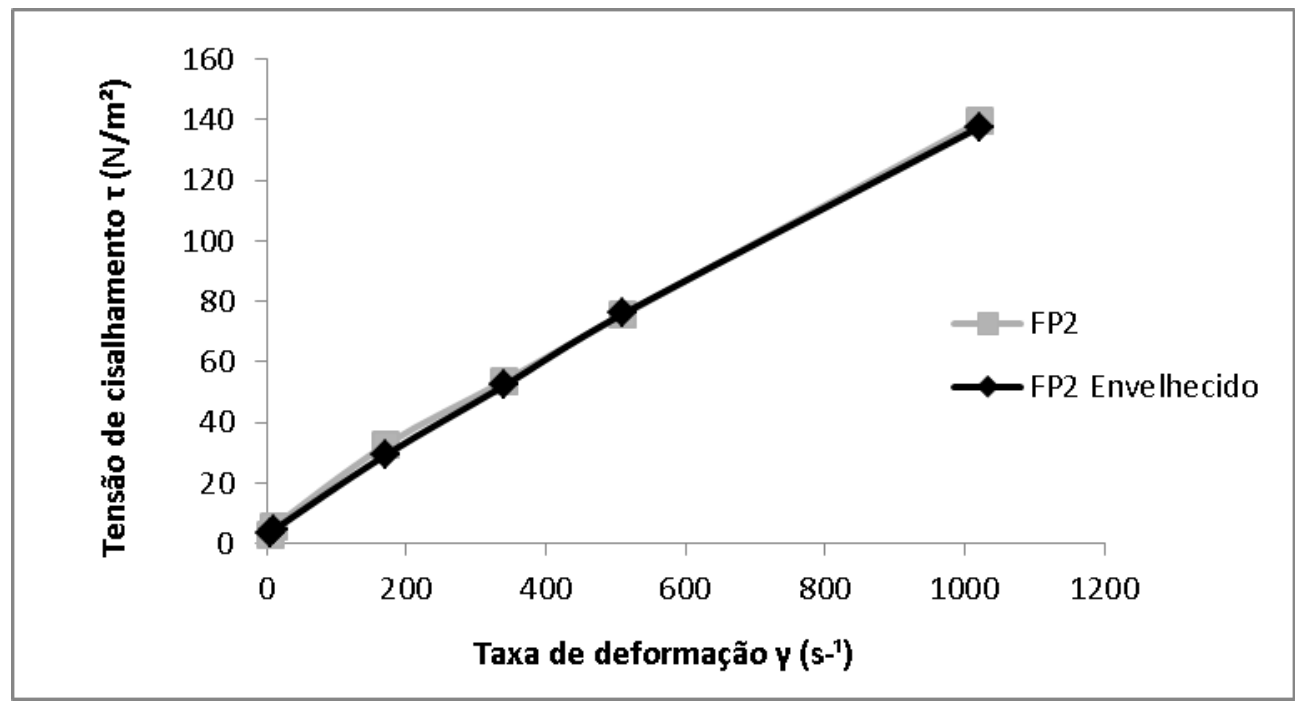

Figura 7: Comportamento reológico da tensão de cisalhamento FP2 e FP2 envelhecido. 
O comportamento reológico da viscosidade é mostrado na Figura 8 e, também, apresentam um comportamento pseudoplástico através da diminuição da viscosidade com o aumento da taxa de deformação $\gamma$.

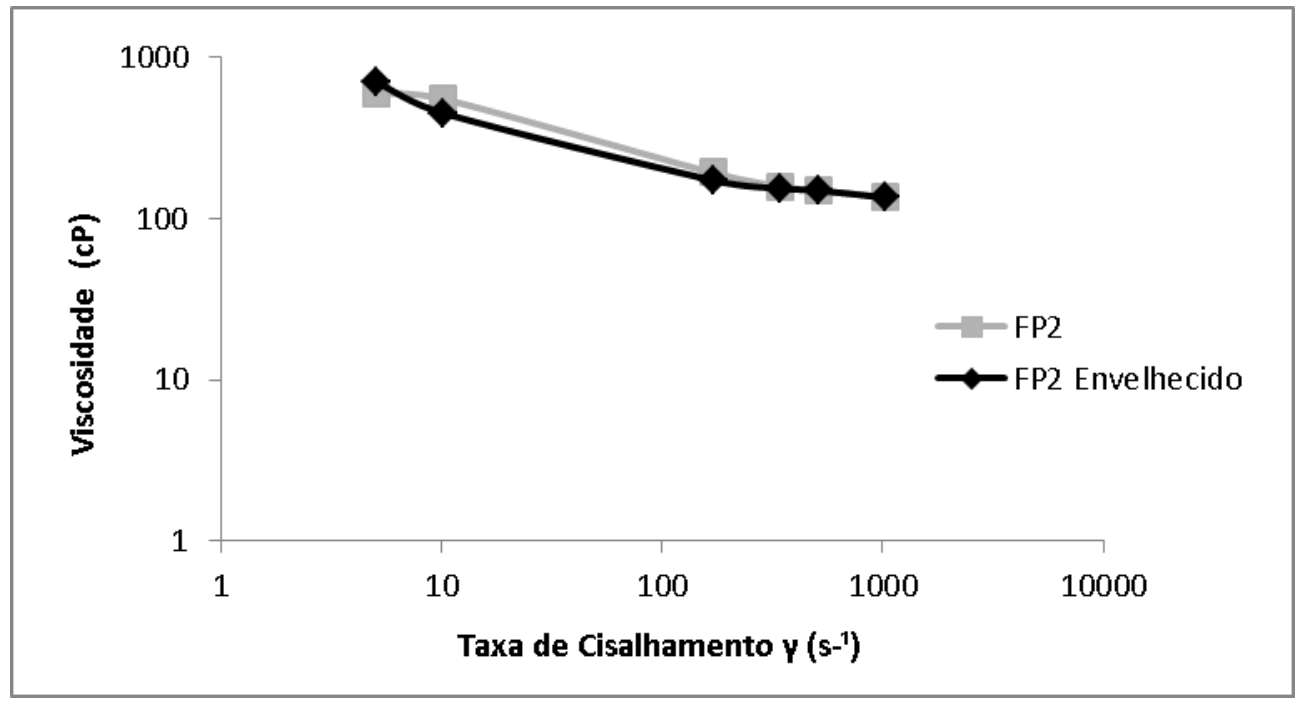

Figura 8: Comportamento reológico da viscosidade FP2 e FP2 envelhecido.

Para a identificação do comportamento não newtoniano, é levado em conta o comportamento entre tensão de cisalhamento $\tau\left(\mathrm{N} / \mathrm{m}^{2}\right)$ e a taxa de deformação $\gamma\left(\mathrm{s}^{-1}\right)$. A observação de uma tensão inicial $\left(\tau_{0}\right)$ como mostrado na Figura 2, associado com uma dinâmica de potência na curva de consistência, denominado de fluido de potência, subsidiam a definição de fluido não Newtoniano, condição essa observada nas curvas obtidas tanto para o FP1 como para o FP2 e, também, nas condições de envelhecimento. Outro fator que contribui para essa definição é o comportamento reológico da viscosidade que, sendo diminuída com o aumento da taxa de cisalhamento, comportamento esse denominado de pseudoplástico, indica uma variação da viscosidade com o tempo, característica inerente a fluidos não newtonianos.

A diminuição da viscosidade à medida que a taxa de cisalhamento aumenta é conhecida como afinamento por cisalhamento e, normalmente, é um comportamento desejável, porque a viscosidade será relativamente baixa em altas taxas de cisalhamento, que prevalecem no tubo de perfuração, reduzindo assim as pressões de bombeamento e, relativamente alta em baixas taxas de cisalhamento, que prevalecem no espaço anular, aumentando assim, a capacidade de carreamento dos cascalhos.

Do estudo reológico, foi possível a determinação dos parâmetros tensão de corte inicial $\left(\tau_{0}\right)$, índice de fluxo (n) e índice de consistência (k), apresentados na Tabela 4.

Tabela 4: Composição de amostras para teste de compatibilidade norma API Spec 10.

\begin{tabular}{c|c|c|c}
\hline FLUIDO & $\left(\tau_{0}\right)\left(\mathrm{N} / \mathrm{m}^{2}\right)$ & $\mathrm{k}\left(\mathrm{Nm}^{-2} \cdot \mathrm{s}^{\mathrm{n}}\right)$ & $\mathrm{n}$ \\
\hline FP1 & 2,05 & 0,19 & 0,922 \\
FP1 Envelhecido & 4,73 & 0,47 & 0,828 \\
FP2 & 3,97 & 0,23 & 0,917 \\
FP2 Envelhecido & 3,05 & 0,24 & 0,914 \\
\hline
\end{tabular}


Os ensaios de compatibilidade entre os fluidos de perfuração FP1 e FP2 e a pasta de cimento padrão resultaram em misturas homogêneas e estáveis. A investigação da reologia dessas misturas proporciona o estudo do comportamento dessa possível mistura em condições de operação, os resultados obtidos a partir de medidas de tensão de cisalhamento $(\tau)$ e taxa de deformação $(\gamma)$ é mostrada na Figura 9.

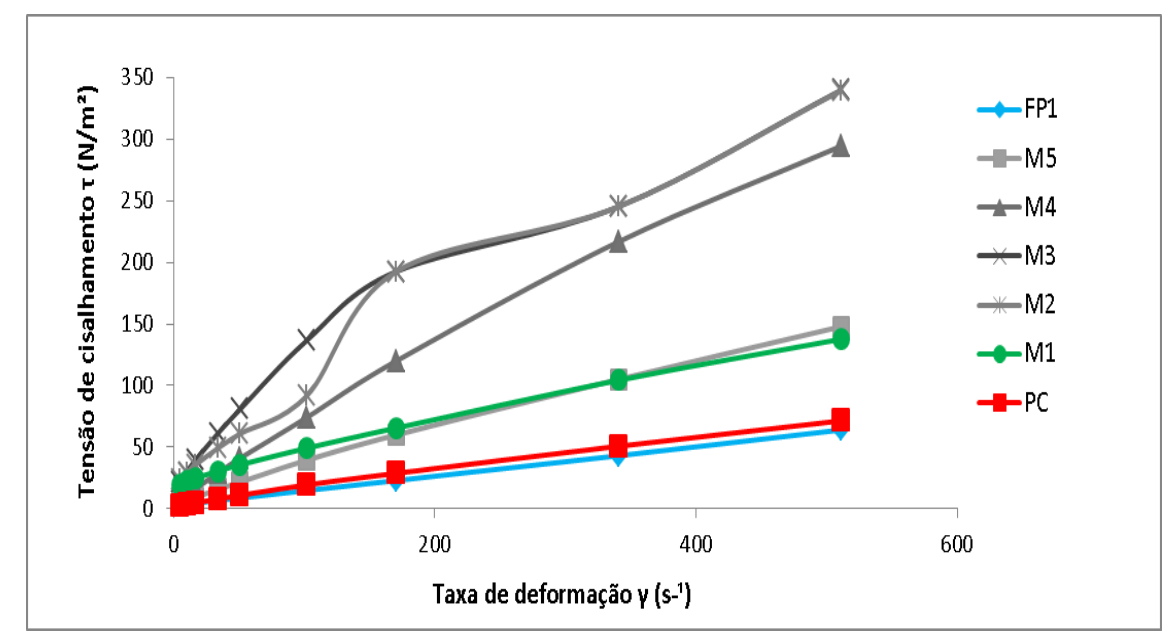

Figura 9: Compatibilidade reológica FP1.

Da Figura 9 é possível observar que, o fluido de perfuração FP1 e a pasta de cimento padrão respondem similarmente a uma taxa de deformação $(\gamma)$, e as misturas $M 1, M 2, M 3, M 4$ e M5 mostram um aumento da Tensão de cisalhamento $(\tau)$.

É importante destacar que a pasta de cimento e o fluido de perfuração estarão em contato apenas em um caso de contaminação, uma vez que no momento da cimentação do poço, o fluido de perfuração, teoricamente, já não deve mais estar presente no poço, sendo assim, a suposta contaminação é estudada aqui nas diferentes proporções das misturas entre pasta de cimento e fluido de perfuração.

As misturas M2, M3 e M4 apresentam um comportamento crítico, tendo seus valores de tensão de cisalhamento $(\tau)$ bem elevados, isso acontece pelo motivo do fluido ser base óleo e a pasta de cimento padrão naturalmente não possuir afinidade a compostos base óleo, comportamento esse também observado no estudo de Soares (2014), sendo ainda possível observar na Figura 10, que a viscosidade dessas misturas, apresenta valores maiores.

A mistura M1 apresenta um cenário que na investigação de um ensaio de contaminação é mais provável de ocorrer, uma vez que possui uma maior proporção de pasta de cimento padrão. Ao se analisar essa mistura, é possível identificar que a tensão de cisalhamento $(\tau)$ se encontra mais próxima aos valores iniciais do fluido de perfuração FP1 e a própria pasta de cimento PC. Já os valores de viscosidade M1 (Figura 10), mais uma vez se alinham com os valores obtidos no FP1 e na PC, sendo dentre todas as misturas a que melhor se adequa a uma possível contaminação, pela proximidade dos valores de referência.

Levando em conta um cenário provável de contaminação em que M1 (95 PC/5 FP1) é a condição investigada, os resultados obtidos são satisfatórios para esse teste de compatibilidade. 


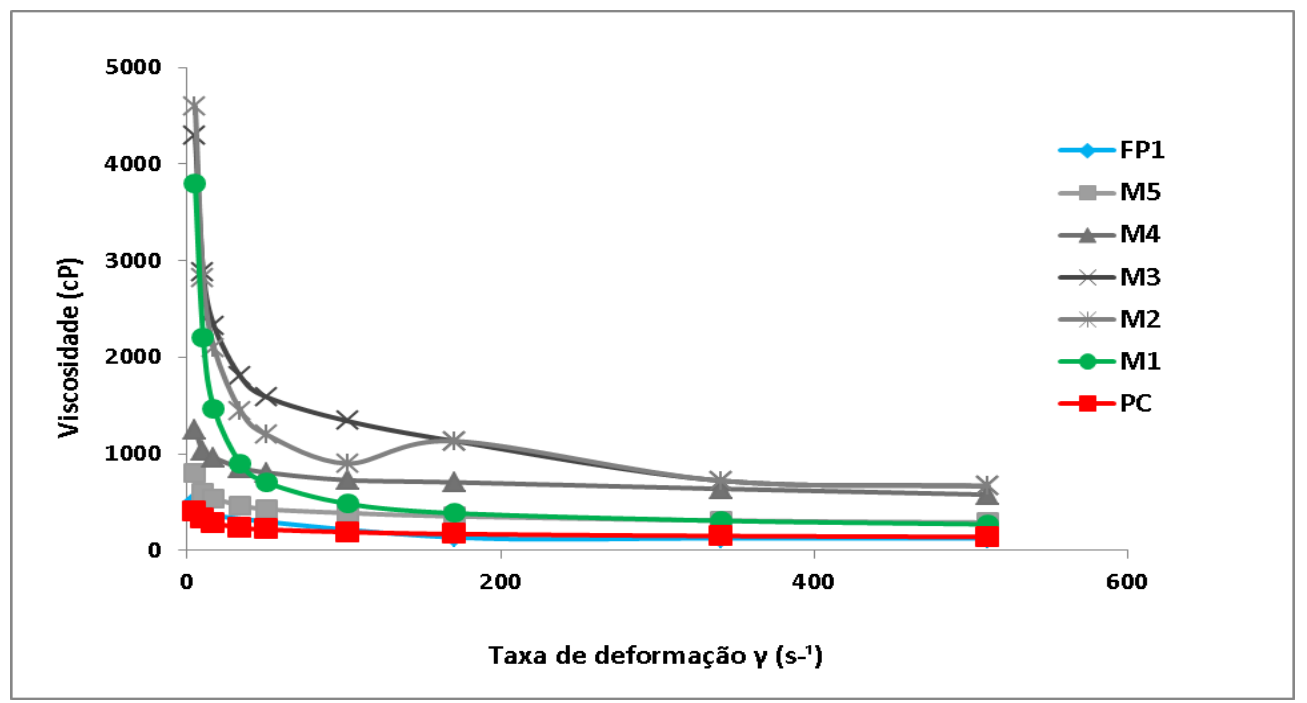

Figura 10: Viscosidade das misturas PC/FP1 no teste de compatibilidade.

Para o fluido de perfuração FP2, os resultados obtidos entre tensão de cisalhamento e taxa de deformação são expostos na Figura 11 e Figura 12.

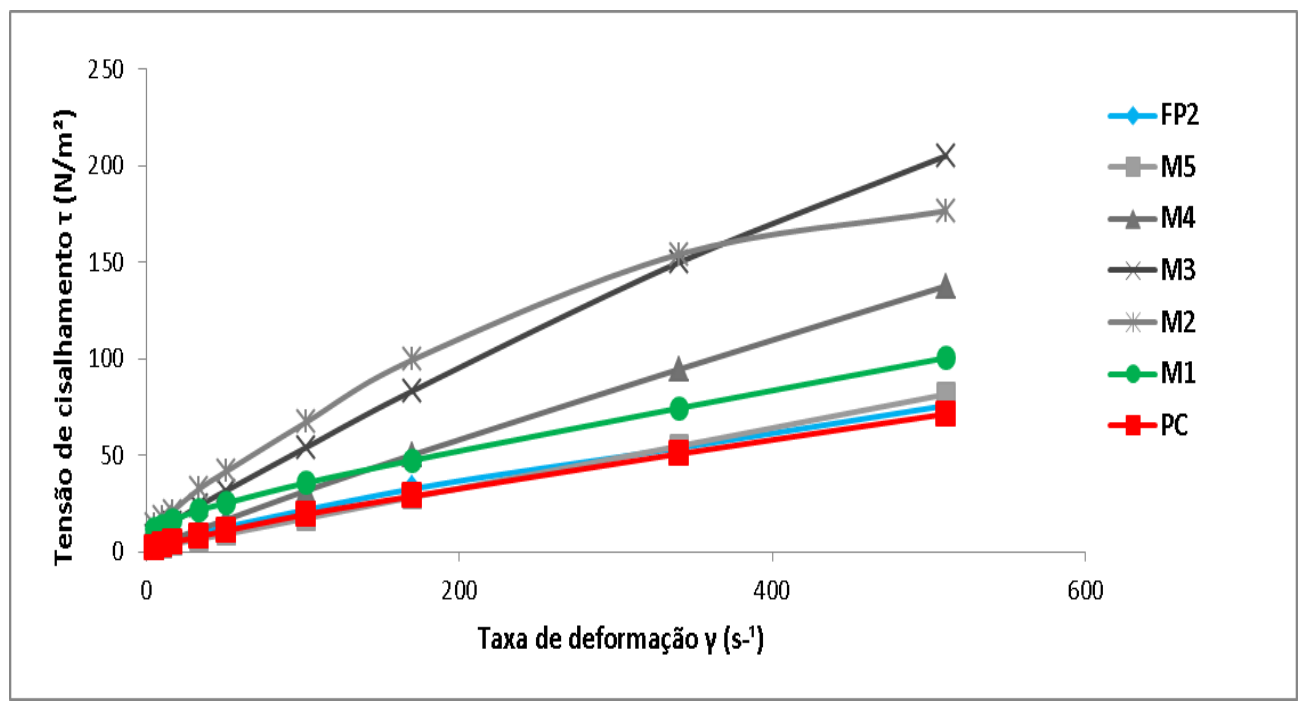

Figura 11: Compatibilidade reológica FP2.

De forma similar ao FP1, o FP2 apresenta uma dinâmica de reposta similar quando submetidos a uma taxa de deformação $(\gamma)$, de forma que para FP2, o intervalo de variação da tensão de cisalhamento $(\tau)$ é menor, tendo as misturas $M 2, M 3$ e M4 com o maior afastamento, já citado anteriormente na análise do FP1.

A mistura M1, também para o fluido de perfuração FP2, apesar de ter uma tensão um pouco maior que os valores de referência que são a pasta de cimento padrão e o fluido FP2, o comportamento e o próprio valor se alinham com os desejados, o que é possível identificar, também, no estudo da viscosidade mostrado na Figura 12. 


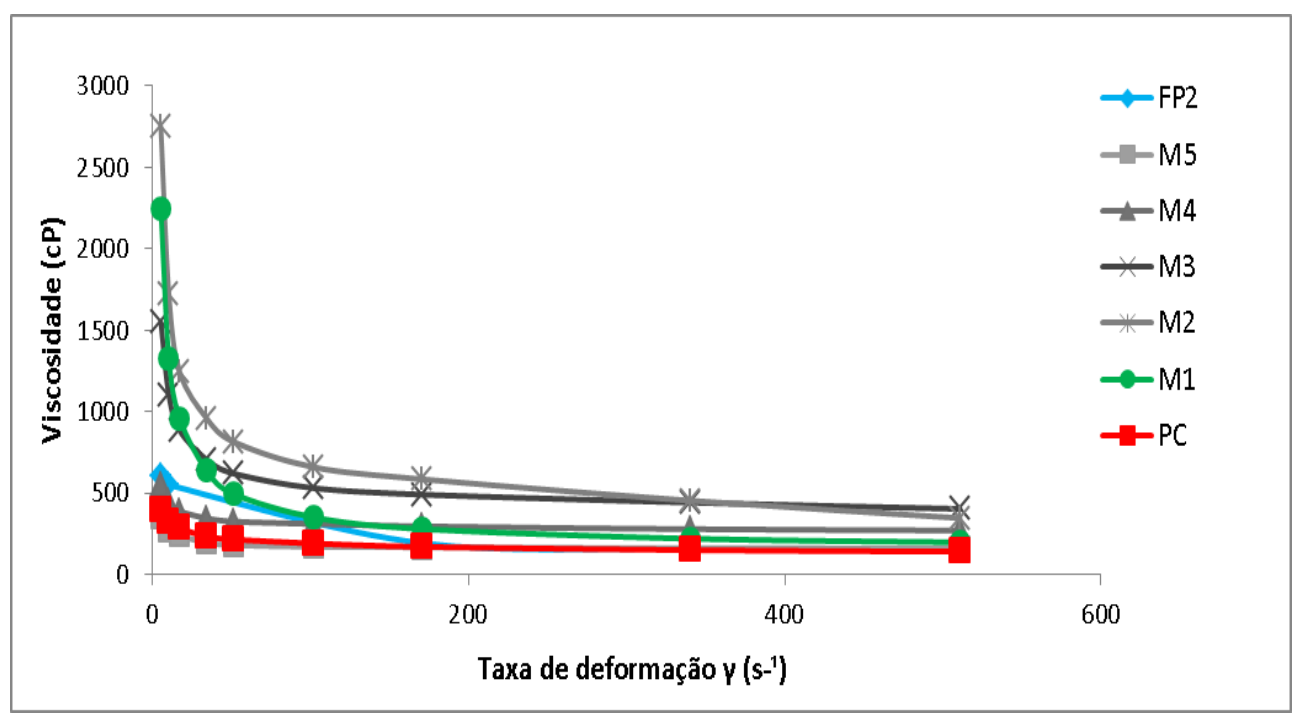

Figura 12: Viscosidade das misturas PC/FP2 no teste de compatibilidade.

Num possível cenário de contaminação, o teste de compatibilidade do FP2 com pasta de cimento, a mistura M1 (95 PC/5 FP1) apresentou resultados satisfatórios.

\section{CONCLUSÃO}

O modelo de Herschel-Bulkley ajustou-se adequadamente aos dados reológicos dos fluidos de perfuração obtidos.

O envelhecimento dos fluidos de perfuração não provocou alterações significativas nas propriedades reológicas, mantendo assim suas funções operacionais.

Os fluidos de perfuração mostraram-se compatíveis com a pasta de cimento, indicando sua eficácia numa possível contaminação.

\section{REFERÊNCIAS BIBLIOGRÁFICAS}

Ribeiro, L., S. (2013). Fluidos de perfuração, completação e estações de fluidos (Trabalho de conclusão de curso). Universidade Federal do Rio Grande do Norte, Natal, RN, Brasil.

Thomas, J.E. (2001). Fundamentos de Engenharia de Petróleo. Rio de Janeiro: Interciência, PETROBRAS.

Son, M. O. (1973). Use of micellar dispersions as drilling fluids. US 1973/3734856A. UNITED STATES PATNT OFFICE.

Davies, S. N.; Meeten, G.H.; Way, P. W. (1973). Water based drilling fluid additive and methods of using fluids containing additives. 5652200. 29 de Julho de 1997. UNITED STATES PATENT. Fluids. 7th Ed., Gulf Publishing Company, Houston, Texas.

Cunha Filho, F. J. V. (2015). Estudo Reológico de um Fluido de Perfuração à Base n-Parafina, Utilizando Argila Modificada com Tensoativo (Dissertação de Mestrado). Universidade Federal do Rio Grande do Norte, Natal, RN, Brasil. 
Sousa, R. P. de. (2018). Otimização e estudo de fluidos de perfuração formulados a partir de microemulsão $O / A$ e $A / O$ (Dissertação de Mestrado). Universidade Federal Paraíba, João Pessoa, PB, Brasil.

Magalhães, R. R. (2018). Obtenção e avaliação da reologia e toxicidade de um fluido de perfuração de base microemulsionada (Dissertação de Mestrado), Universidade Federal da Paraíba, João Pessoa, PB, Brasil.

Silva, G. C. (2011). Sistema microemulsionado: caracterização e aplicação na indústria de petróleo (Tese de Doutorado). Programa de Pós-Graduação em Química, Universidade Federal do Rio Grande do Norte, Natal, RN, Brasil.

Hayes, J. B.; Haws, G. W.; Gogarty, W. B. (1997). Water-in-oil microemulsion drilling fluids. 4,012,329. 14 de Março de 1977. UNITED STATES PATENT.

Quintero, L.; Jones, T. A.; Clark, D. E.; Schwertner, D. (2009). Cases History Studies of Production Enhancement in Cased Hole Wells Using Microemulsion Fluids. Society of Petroleum Engineers. doi:10.2118/121926-MS

Amorim, L. V. (2003). Melhoria, proteção e recuperação da reologia de fluidos hidroargilosos para uso na perfuração de poços de petróleo (Tese de Doutorado). Universidade Federal de Campina Grande, Campina Grande, PB, Brasil.

Campos, G. (2005). Procedimentos e métodos de laboratório destinados a cimentação de poços petrolíferos.

Asme, S. S. C. (2005). Drilling fluids processing handbook. Burlington, USA: Gulf Professional Publishing.

Caenn, R.; Chillingar, G. V. (1996). Drilling Fluids: State of the Art. Journal of Petroleum Science and Engineering, (14, pp. 221-230).

Darley, H. C. H.; Gray, G. R. (2017). Composition and Properties of Drilling and Completion Fluids. 7th Ed., Gulf Publishing Company, Houston, Texas.

Machado, J. C. (2002). Fundamentos e Classificação de Fluidos Viscosos. In: Reologia e Escoamento de Fluidos: Ênfase na indústria do petróleo. Rio de Janeiro: Interciência.

Shiroma, P. H. (2012). Estudo do comportamento reológico de suspensão aquosa de bentonita e CMC: influência da concentração do $\mathrm{NaCl}$ (Dissertação de Mestrado). Escola Politécnica da Universidade de São Paulo, São Paulo, SP, Brasil.

Norma API 13B, de 2012. Recommended Practice for Field Testing Oil-Based Drilling Fluids. API Recommended Practice 13B-2.

Petrobras, (1988). Viscosificantes para Fluido de Perfuração Base de Água na Exploração e Produção de Petróleo, Especificação, N 2604. 
Soares, A. A. (2014). Avaliação de compatibilidade entre pasta de cimento e fluido de perfuração não-aquoso utilizados em poços petrolíferos (Dissertação de Mestrado). Universidade Federal do Rio Grande do Norte, Natal, RN, Brasil.

10A, A. S. (2004). API specification for materials and testing for well cements. Washington DC: Americam Petroleum Inst.

\section{COMO CITAR ESTE ARTIGO:}

Garnica, A. I. C., Soares, A. S. L., Curbelo, F. D. DA S. (2020). Estudo reológico de um fluido de perfuração com características biodegradáveis a base de tensoativo NP40 e óleo de canola. Holos. 36(6), 1-15.

\section{SOBRE OS AUTORES}

\section{A. I. C. GARNICA}

Formado em Engenharia Química pela Universidade Central de Las Villas, Marta Abreu/Villa Clara/Cuba. Fez mestrado e doutorado em Engenharia Química no PPGEQ/UFRN. Atualmente, é Professor associado IV do Departamento de Engenharia Química/DEQ/UFPB e trabalha em pesquisas na área de petróleo com ênfase em fluidos de perfuração, colchões lavadores, lubrificantes e tratamento de efluentes de esta indústria. Também, trabalha no projeto de equipamentos visando, principalmente, as operações de destilação, absorção, adsorção e extração líquido-líquido. Possui artigos e patentes depositadas nas áreas de atuação mencionadas anteriormente. E-mail: alfredocurbelo@yahoo.com

ORCID ID: http://orcid.org/0000-0001-9600-5110

\section{A. S. L. SOARES}

Formado em Engenharia Química pela universidade de Federal da Paraíba, é mestrando do programa de Pósgraduação em Engenharia Química PPGEQ/DEQ/UFPB. Está desenvolvendo sua dissertação na área de petróleo e gás natural. E-mail: andersonshimenes@hotmail.com

ORCID ID: https://orcid.org/0000-0002-9363-3899

\section{F. D. DA S. CURBELO}

Formada em Engenharia Química pela Universidade de Federal do Rio Grande do Norte (UFRN). Fez mestrado e doutorado em Engenharia Química no PPGEQ/UFRN. Atualmente é Professora Associado II do Departamento de Engenharia Química/DEQ/UFPB e trabalha em pesquisas na área de tensoativos e de petróleo com ênfase em fluidos de perfuração, colchões lavadores, recuperação avançada de petróleo, lubrificantes e tratamento de efluentes. Também trabalha no projeto de equipamentos visando, principalmente, as operações de destilação, absorção, adsorção e extração líquido-líquido. Possui artigos e patentes depositadas nas áreas de atuação mencionadas anteriormente. E-mail: fabioladias@yahoo.com ORCID ID: http://orcid.org/0000-0002-1501-5702

Editor(a) Responsável: Francinaide de Lima Silva Nascimento

Pareceristas Ad Hoc: Leandro Correia e George Simonelli

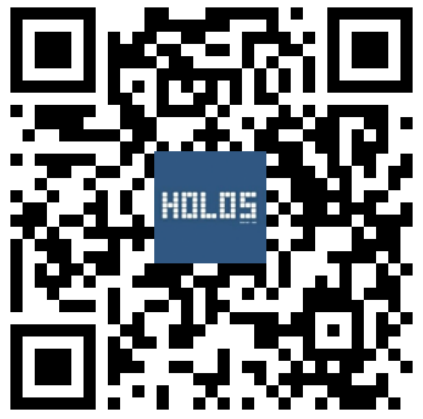

